\title{
Objective-lens-free confocal endomicroscope using Lissajous scanning lensed-fiber
}

\author{
Jae-Beom Kim, ${ }^{\text {a,b, }, \dagger}$ Jaehun Jeon $\odot,{ }^{\text {a,b, }, \dagger}$ Kyungmin Hwang, ${ }^{\text {a,b }}$ \\ Daniel Y. Kim, ${ }^{\text {a,b }}$ and Ki-Hun Jeong ${ }^{a, b}, *$ \\ ${ }^{a}$ Korea Advanced Institute of Science and Technology, \\ Department of Bio and Brain Engineering, Daejeon, Republic of Korea \\ ${ }^{b}$ KAIST Institute for Health Science and Technology, KAIST, Daejeon, Republic of Korea
}

\begin{abstract}
We report an objective-lens-free endomicroscopic catheter for compact Lissajous scanned confocal endomicroscopic system using a scanning lensed-fiber. The fiber scanner comprises a single-mode fiber (SMF) spliced with a coreless silica fiber segment and quadrupole piezoelectric tube. An objective lens was directly formed at the distal end of an SMF using a fusion splicer. The lensed fiber eliminates the need for conventional beam focusing elements such as a gradient index rod lens and precise optical alignment, allowing low cost and facile fabrication of an exceptionally short endomicroscopic catheter. The endomicroscopic scanner was fully packaged within a stainless tube of $2.6 \mathrm{~mm}$ in outer diameter and $20 \mathrm{~mm}$ in length, which can be easily inserted through the working channel of conventional laparoscope or colonoscope. The microscopic images of a stained kidney section and ear of a mouse were successfully obtained with the Lissajous scanning confocal endomicroscope. The compact Lissajous scanning lensed fiber can provide a new route for diverse in vivo endomicroscopic applications. (C) The Authors. Published by SPIE under a Creative Commons Attribution 4.0 Unported License. Distribution or reproduction of this work in whole or in part requires full attribution of the original publication, including its DOI. [DOI: 10.1117/1.JOM.1.3.034501]
\end{abstract}

Keywords: Lissajous scanning; endomicroscope; lensed-fiber; endomicroscopic scanner.

Paper 21004 received Feb. 8, 2021; accepted for publication May 14, 2021; published online May 28, 2021.

\section{Introduction}

Endomicroscopes are actively combined with advanced imaging techniques such as multiphoton microscopy,${ }^{1-4}$ optical coherence tomography (OCT),${ }^{5-7}$ and confocal microscopy,${ }^{8-10}$ and they allow minimally invasive endoscopic diagnosis and in vivo imaging. Despite the great potential, the integration of micro-optical modules inside an endomicroscope is still challenging due to their bulk size and lack of conventional optical components. A fiber-optic scanning probe plays an important role for developing compact endomicroscopic imaging probes due to many technical benefits such as compactness, flexibility, lightweight, cost effectiveness, and biocompatibility. In particular, compact fiber-optic probes facilitate the direction insertion through the working channel of an endoscope for on-demand detection and real-time diagnosis.

In recent years, assorted micro-optic probes have been extensively incorporated for endomicroscopic imaging applications. Conventional endomicroscopic probes consist of microscanner, optical fiber, gradient index (GRIN) lens, and additional optical elements to scan a laser beam into the region of interest. For instance, microelectromechanical system (MEMS) lens scanning probe,${ }^{6}$ scanning MEMS mirror-based optical probe, ${ }^{11-13}$ or a tubular piezoelectricbased scanning optical fiber ${ }^{14-18}$ allow the compact integration of endomicroscope for clinical applications. In addition, fiber optic probes have been even successfully integrated within a single medical needle. ${ }^{19}$ However, conventional probes still require additional optical elements for beam focusing or beam deflection such as prism, mirror, or objective lens. The optical elements require precise optical alignments and large packaging size. A GRIN rod lens often serves as an

\footnotetext{
*Address all correspondence to Ki-Hun Jeong, kjeong@kaist.ac.kr

${ }^{\dagger}$ These authors equally contributed to this work.
} 
objective lens of the probe to minimize the catheter size as well as to increase the image resolution; however, it exhibits some disadvantages such as long rigid length and high manufacturing cost. Moreover, the GRIN lens restricts the numerical aperture (NA) and the field-of-view of an endomicroscopic probe. As a result, a compact and alignment-free packaging of an imaging probe is still in need for clinical endomicroscopic applications.

A scanning lensed fiber can provide a simple solution for compact packaging of alignmentfree laser scanning applications. ${ }^{20-22}$ Lissajous scanning or biaxial resonant scanning has been employed for fiber-scanning endomicroscopes to improve the mechanical stability and the scanning uniformity. ${ }^{23,24}$ Recently, the selection rule for scanning frequencies realizes high-definition and high frame rate (HDHF) for Lissajous scanned imaging. ${ }^{25}$ However, compact optical packaging is still challenging in the integration of scanning fiber-based endomicroscopic probes, which can be physically applicable for conventional clinical endoscopes.

Here, we report an objective-free optical fiber scanner for confocal endomicroscope using Lissajous scanning lensed fiber. Figure 1(a) shows the schematic illustration of confocal endomicroscopic probe, which comprises a quadrupole piezoelectric (PZT) fiber scanner and a single-mode fiber (SMF) serially concatenated with a coreless silica fiber (CSF) segment. The lens was made by melting at the end of CSF using a fusion splicer, which focused incident light source to the target. The length of the CSF and the radius of curvature (ROC) of the lens determined the imaging characteristics such as working distance and beam spot size. The ROC of the

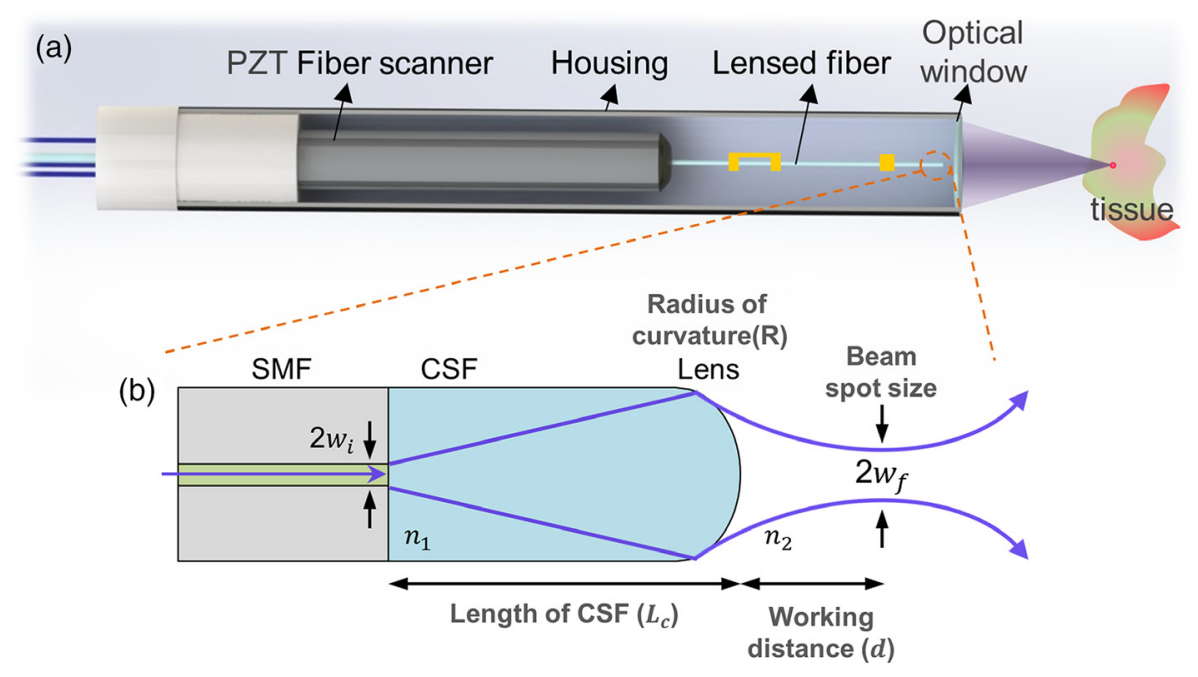

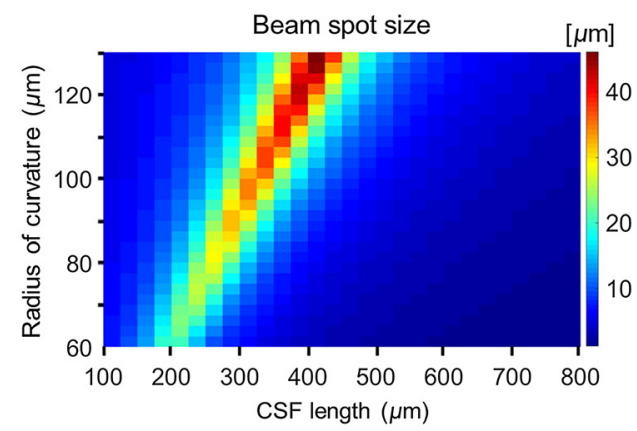

(c)

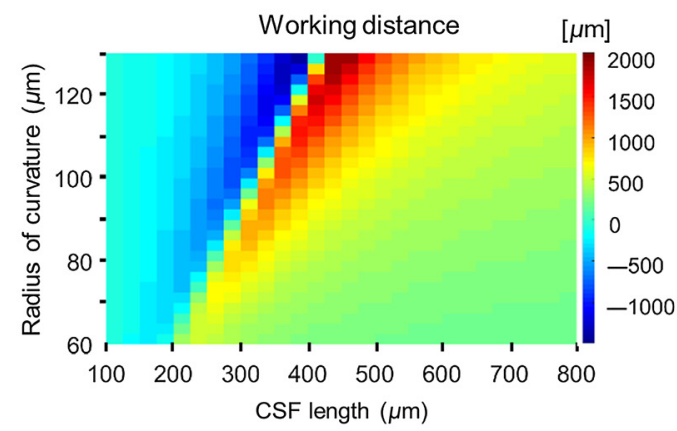

(d)

Fig. 1 (a) Illustration of the Lissajous scanning lensed fiber for confocal endomicroscope. The compact endomicroscopic probe comprises a PZT fiber scanner and a lensed fiber. (b) Design parameters of the lensed fiber. The lensed fiber consists of SMF, CSF, and a built-in lens at the end of CSF. The incident beam from the SMF is expanded through the CSF region and focused by a fiber lens. (c), (d) Numerical simulation results of lensed fiber. (c) The beam spot size and (d) the working distance of the lensed fiber depend on the length of CSF and the ROC of the fiber optic lens. 
lensed fiber was controlled by filament power and duration time. This all-fiber scanner allows no need for additional optical elements such as GRIN lens for focusing light and precise alignment. The Lissajous lensed fiber scanner was fully packaged with $2.6 \mathrm{~mm}$ diameter as an endomicroscopic probe and coupled with a confocal imaging system.

Figure 1(b) shows the design schematics of lensed fiber. The lensed fiber consists of a hybrid optical fiber comprising an SMF serially fusion spliced with a CSF segment and a built-in lens fabricated at the end of CSF. The CSF as a beam expanding region was applied to achieve a sufficiently large beam size to the lens. The curved surface of the lens focuses incident light to the sample and collects photons from the sample for confocal imaging. The optical performance of the lensed fiber was analyzed using the ray matrix transformation, based on the ABCD matrix analysis at 488 -nm wavelength. ${ }^{26,27}$ Light propagation was considered as a Gaussian beam in this theoretical model. The working distance $d$ and the beam waist size $\omega_{f}$ of a lensed fiber are described as

$$
\begin{gathered}
d=\frac{\left(\frac{n_{1}-n_{2}}{n_{2} R}\right)-\left(\frac{\lambda}{n_{1} \pi \omega_{i}^{2}}\right)^{2} L_{c}\left(\frac{n_{1}}{n_{2}}+\frac{L_{c}\left(n_{2}-n_{1}\right)}{n_{2} R}\right)}{\left(\frac{n_{2}-n_{1}}{n_{2} R}\right)^{2}+\left(\frac{\lambda}{n_{1} \pi \omega_{i}^{2}}\right)^{2}\left(\frac{n_{1}}{n_{2}}+\frac{L_{c}\left(n_{2}-n_{1}\right)}{n_{2} R}\right)^{2}}, \\
\omega_{f}=\omega_{i} \sqrt{\left(1+\frac{n_{2}-n_{1}}{n_{2} R} d\right)^{2}+\left(\frac{\lambda}{n_{1} \pi \omega_{i}^{2}}\right)^{2}\left(\frac{n_{1}}{n_{2}} d+L_{c}\left(1+\frac{n_{2}-n_{1}}{n_{2} R} d\right)\right)^{2}} .
\end{gathered}
$$

The terms of $n_{1}, n_{2}, R, \lambda, \omega_{i}$, and $L_{c}$ denote the refractive index of CSF (1.4630 at $488 \mathrm{~nm}$ in wavelength), the refractive index of the air, the ROC of a lens, a light wavelength $(488 \mathrm{~nm})$, the beam radius of SMF ( $\left.\omega_{i}=3.7 \mu \mathrm{m}\right)$, the length of CSF, respectively. The length of the CSF and the ROC serve as the critical design factors for the working distance and the beam waist size. The commercial SMF and CSF were employed in the simulations and the input Gaussian beam was $488 \mathrm{~nm}$ wavelength. Figures 1(c) and 1(d) show the calculated results for the beam spot size and the working distance depending on the CSF length and the ROC of a lens. A wide range of imaging characteristics can be determined by properly selecting the design parameters. Note that, a small beam spot size was desirable for obtaining high-resolution confocal imaging. The calculated results clearly indicate that the minimum beam spot at focus occurs when the CSF length becomes longer with the small ROC of a fiber lens.

\section{Results and Discussion}

Figure 2(a) outlines the fabrication process for the lensed fiber probe includes three main steps. A 125- $\mu \mathrm{m}$ diameter SMF (S405XP, Thorlabs, Inc.) and CSF (FG125LA, 125- $\mu$ m cladding diameter, Thorlabs, Inc.) were selected for the fabrication. All protective coatings of fibers were removed in isopropanol alcohol solution prior to the fusion splicing process. The CSF was first fusion-spliced to the distal end of SMF using a fiber-optic fusion splicer (GPX 3400, Vytran) with filament (V2 filament for $125-\mu \mathrm{m}$ diameter optical fiber). An optical fiber cleaver (LDC400, Vytran) was then employed to obtain desire length of CSF under the precise control of cleave position. Afterward, the SMF spliced with certain length of CSF was placed in fusion splicer to fabricate a lens at the end of CSF. The incident light source exits from the SMF core expands through CSF region. Note that, the CSF was employed to achieve a sufficiently large beam size to lens. On the other hand, concentric interference patterns generate after a certain length of CSF, which are due to reflection of light from the CSF-air boundary. Therefore, CSF length was first optimized considering the NA of SMF, refractive index of CSF, and incident wavelength. The optimal length of CSF was selected about $650 \mu \mathrm{m}$ by measuring the beam propagation using confocal laser scanning microscopy (LSM 510/CF26, Carl Zeiss). The lens for beam focusing was fabricated by melting the end of CSF after forming the beam expanding region. The ROC of the lensed fiber was controlled by filament power with fixed duration time of fusion splicer. The ROC at $62.5 \mu \mathrm{m}$ away from the axial axis can be achieved from 75 to $180 \mu \mathrm{m}$ with different filament power as shown in Fig. 2(b). A low electric discharge power may cause an 


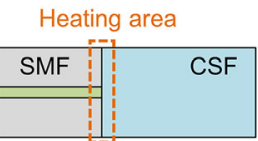

(i) Fusion splicing

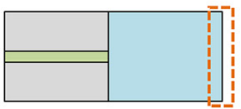

(iii) Melting

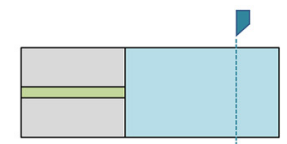

(ii) Cleaving

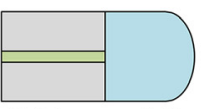

(iv) Lensed fiber

(a)

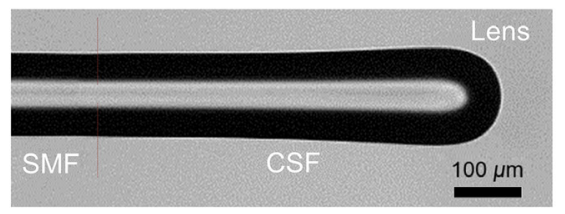

(c)

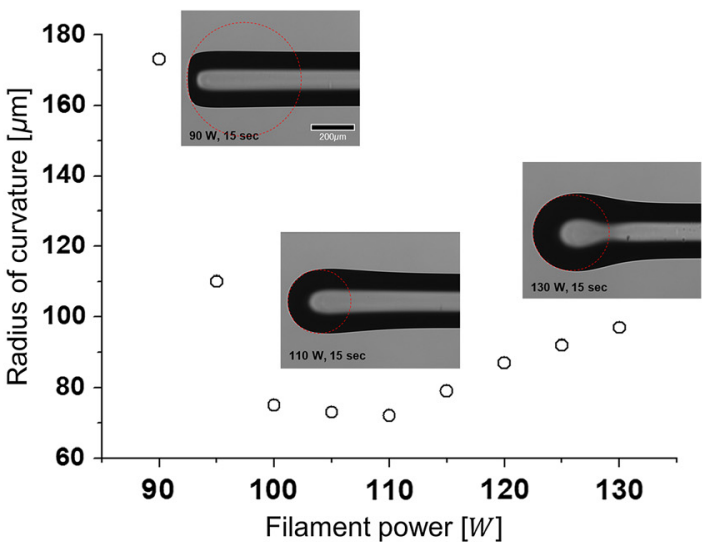

(b)

Fig. 2 (a) Fabrication procedure of lensed fiber. (b) The filament power of fusion splicer was the parameter to control the ROC of the lens. (c) Optical image of a fabricated lensed fiber.

incompletely melted CSF, which results in the large value and the variation of ROC. However, a longer melting time under high power results in low ROC and low variation. Under applied higher power, the ROC variation along the lateral direction increases due to the gravity and then the melted CSF further increases the ROC. The minimum ROC of $75 \mu \mathrm{m}$ with $650 \mu \mathrm{m} \mathrm{CSF}$ length was fabricated to achieve a high-resolution confocal imaging probe as shown Fig. 2(c). The measured ROC of upper and lower hemisphere shows only $0.4 \%$ difference and the maximum ROC difference along the axial axis is measured by $9 \mu \mathrm{m}$. The working distance and focused beam spot size were evaluated using a confocal laser scanning microscope and 488-nm fiber coupled laser source. The incident beam propagating through the SMF was expanded in the CSF region and then focused by the fiber lens. The calculated NA and the working distance of the lensed fiber are 0.16 and $290 \mu \mathrm{m}$, respectively. In addition, the full-width half-maximum (FWHM) of the focal spot is $2.3 \mu \mathrm{m}$.

A fully packaged confocal endomicroscope was developed using a Lissajous lensed fiber scanner. A lensed fiber was assembled with PZT-driven optical fiber scanners for Lissajous scanning as shown in Fig. 3(a). The length and the diameter of the PZT were 13 and $1.5 \mathrm{~mm}$, respectively. Note that the PZT tube-driven resonant Lissajous fiber scanner is widely used in endomicroscopic catheters, which has many advantages such as high scan amplitude with low operating voltage, compact, and robust packaging. The optical fiber scanner consists of piezoelectric tube, a lensed fiber, and a micromachined tethered silicon oscillator (MTSO). The MTSO consists of a silicon spring and additional mass ${ }^{28}$ and mounted on the lensed fiber with UV curable polymer (NOA 63, Norland Optics) to modulate the stiffness and resonant scanning frequency. The lensed fiber was forced to oscillate in resonant frequency by Lissajous fiber scanner. The fiber scanner operates in first-mode vibration and the resonant frequency of $x$-axis and $y$-axis are 1002 and $1210 \mathrm{~Hz}$, respectively. ${ }^{29}$ The Lissajous scanning lensed fiber was then fully packaged with a stainless-steel tube housing of $2.6 \mathrm{~mm}$ in diameter and $20 \mathrm{~mm}$ in length and an optical window of $2.5 \mathrm{~mm}$ diameter as shown in Figs. 3(b) and 3(c). Note that such a short length of catheter stainless-steel tube allows the direction insertion through the working channel of conventional clinical flexible endoscope. The optical characterization of the probe was also performed by the optical sectioning along the $z$-axis using a conventional confocal laser scanning microscope. The working distance was about $190 \mu \mathrm{m}$ from the optical window and the FWHM of the intensity field at the focal spot is $2.6 \mu \mathrm{m}$ as shown in Figs. 3(d) and 3(e). A slight expansion in FWHM is due to the misalignment during the packaging process. The compactly packaged confocal endomicroscope was easily inserted into the instruments channel of a conventional endoscope such as flexible and rigid endoscope, which can also provide a compact handheld probe for in vivo tissue imaging or optical biopsy applications as shown in Figs. 3(f) and $3(\mathrm{~g})$. 


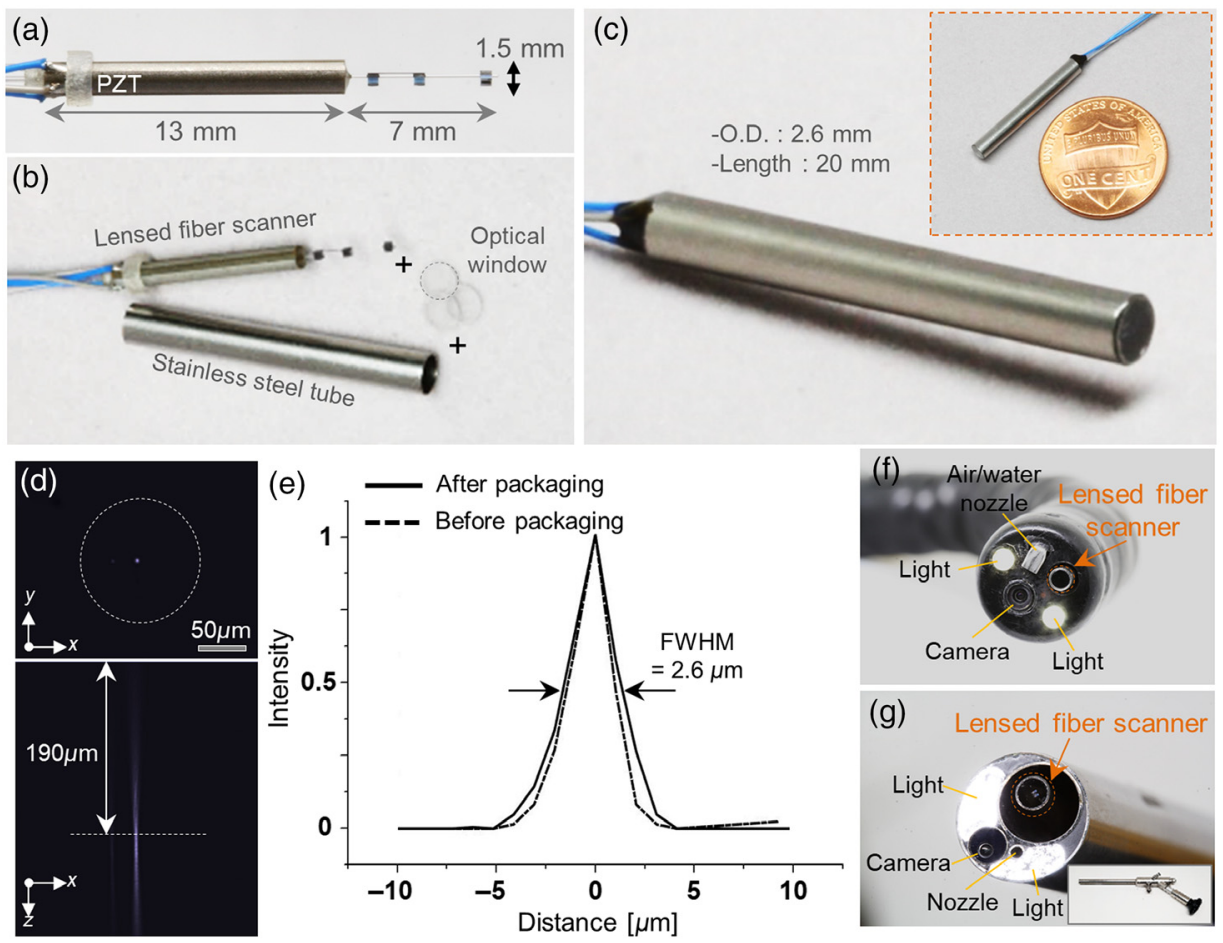

Fig. 3 (a) Lissajous fiber scanner was demonstrated based on a lensed fiber probe with a piezoelectric driven actuator. (b), (c) The Lissajous lensed fiber scanner was fully packaged with a stainless-steel tube (2.6-mm outer diameter and 20-mm total length) and a laser cut cover glass (0101030, Marienfeld) as optical window (2.5-mm outer diameter and 0.14-mm thickness) for developing endomicroscopic probe. (d) Optical characterization of confocal endomicroscopic probe. The beam profile was measured by optical sectioning along the $z$-axis with a conventional confocal laser scanning microscope. (e) The beam profile of probe before (dotted line) and after (solid line) packaging at the focal plane. The working distance is about $190 \mu \mathrm{m}$ from the optical window. The FWHM of the intensity field at the focal spot before and after packaging are 2.3 and $2.6 \mu \mathrm{m}$, respectively. (f), (g) The compact confocal endomicroscopic probe was easily incorporated into an instrument channel of conventional endoscope such as (f) colonoscope and (g) laparoscope.

The fully packaged probe was finally connected to a confocal endomicrscope system. Figure 4(a) shows a schematic illustration for confocal endomicroscope system. The 488-nm wavelength fiber coupled laser source (LDM series 488, LASOS) was coupled to FC/APC collimator (PAFA-4-B, Thorlabs, Inc.) for fluorescein isothiocyanate (FITC) imaging. The excitation light was reflected to the dichroic mirror (DM, FF497-Di01, Semrock) and coupled to the Lissajous scanning lensed fiber probe. The fluorescence signal transmits though the DM and a band pass filter (FF02-525/40-25, Semrock). The photomultiplier tube (PMT: 9110, Hamamatsu) detects fluorescence signals, which was transmitted to an image signal processor (ISP). The ISP consists of a function generator, analog-to-digital convertors (ADC), and a video memory. The ADC converts the analog PMT and driving signals to digital signal with $2.5-\mathrm{MHz}$ sampling rate. The phase delay between the driving signal and the fiber scanner motion was matched manually, and adjusted digital signals were transferred to the video memory. The ISP reconstructs image coordinates by mapping the PMT signal based on the calibrated driving signals in the confocal images of $256 \times 256$ pixels on the field programmable gate array board. The reconstructed image is finally displayed on the monitor. The system provides $10-\mathrm{Hz}$ frame rate and over $80 \%$ fill factor by operating the fiber scanner with $1000-$ and $1210-\mathrm{Hz}$ resonant frequencies. Using the selection rule of scanning frequencies, both axis scanning frequencies are selected to have high greatest common devisor (GCD) and high total lobe number for Lissajous pattern, i.e., the sum of scanning frequencies divided by the GCD of biaxial scanning frequencies. ${ }^{25}$ The confocal fluorescence images were obtained from a stained mouse kidney 


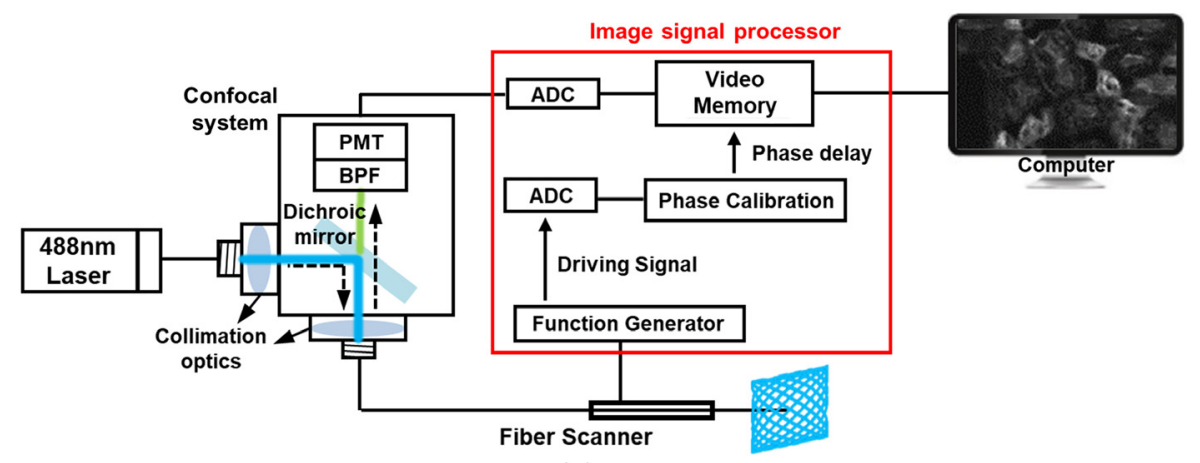

(a)

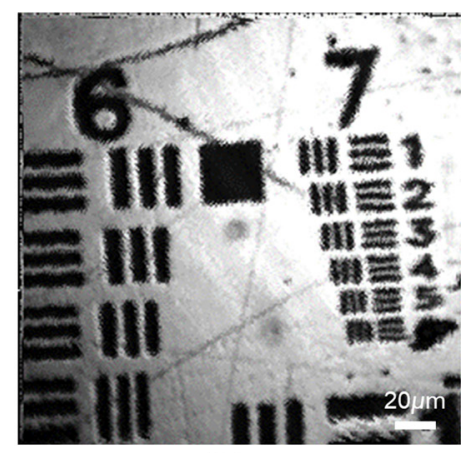

(b)

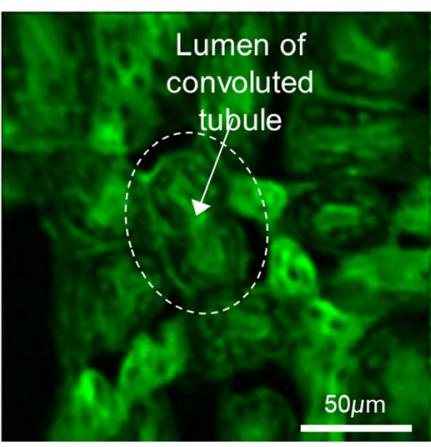

(c)

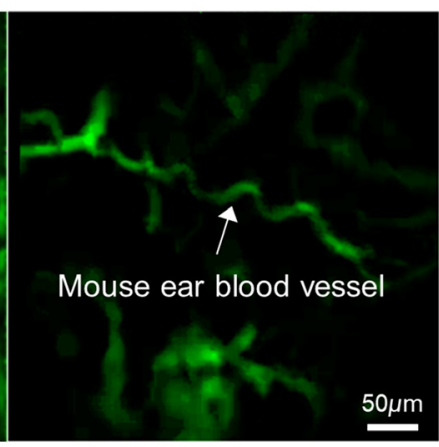

(d)

Fig. 4 (a) A schematic illustration for confocal endomicroscope system. (b) Confocal reflectance images of groups 6 and 7 from high-resolution USAF test target acquired using the Lissajous lensed fiber scanner. (c) The ex vivo image of mouse kidney section stained with Alexa Fluor 488 (F-24630, Invitrogen) using confocal endomicroscope system. The lumen of the convoluted tubule is marked with white arrows. (d) An ex vivo confocal fluorescence image of mouse ear blood vessel after injecting 1\% FITC to a wild-type mouse. The total FOV and applied voltage of each figures were (b) $217 \mu \mathrm{m} \times 217 \mu \mathrm{m}$ at $\sim 30 V_{\mathrm{pp}}$, (c) $194 \mu \mathrm{m} \times 194 \mu \mathrm{m}$ at $\sim 30 V_{\mathrm{pp}}$, and (d) $428 \mu \mathrm{m} \times 428 \mu \mathrm{m}$ at $\sim 50 V_{\mathrm{pp}}$.

section and mouse ear skin to evaluate the imaging performance of Lissajous scanning lensed fiber. Figure 4(b) shows the reflectance imaging result of groups 6 and 7 in aluminum pattern of US Air Force (USAF) target. The minimum width of about $2.19 \mu \mathrm{m}$ in group 7 was clearly resolved by confocal endomicroscope. The total FOV was $217 \mu \mathrm{m} \times 217 \mu \mathrm{m}$ at $30 V_{\mathrm{pp}}$. An ex vivo imaging of the mouse kidney section stained with Alexa Fluor 488 wheat germ agglutinin was performed to compare confocal images using endomicroscope system and conventional confocal laser scanning microscope as shown in Fig. 4(c). The convoluted tubules of the mouse kidney were clearly observed in confocal images, which was marked with white arrows. The ex vivo confocal fluorescence imaging while in contact with mouse ear blood vessel was also performed as shown in Fig. 4(d). The confocal images are comparable to those from GRIN lensbased fiber scanning endomicroscope, ${ }^{29}$ although the lensed fiber endomicroscopic probe is fully packaged with $30 \%$ shorter length. The confocal fluorescence imaging results of biological specimen show that the developed compact Lissajous scanning lensed fiber probe can be replaced conventional bulk-optics-based imaging probe.

\section{Conclusions}

In summary, this work has successfully developed the objective lens-free imaging probe for confocal laser endomicroscope. The Lissajous scanning lensed fiber was developed to eliminate bulk beam focusing elements and demonstrated confocal imaging of a biological specimen. The ray tracing analysis using $\mathrm{ABCD}$ matrix was performed to optimize the imaging characteristics of the lensed fiber. The lensed fiber scanner was fully packaged with a stainless-steel tube 
housing of $2.6 \mathrm{~mm}$ in diameter and $20 \mathrm{~mm}$ in length. The compact scanning probe was easily incorporated into a conventional endoscope such as colonoscope and laparoscope. The reflection and ex vivo confocal images were successfully obtained using confocal endomicroscope without conventional GRIN lens-based optical system. This compact Lissajous scanning lensed fiber can provide a new direction for developing miniaturized endoscopic imaging probe such as OCT, confocal laser microscopy, or two-photon microscopy.

\section{Acknowledgments}

This work was supported by the National Research Foundation of Korea (NRF) funded by the Ministry of Science ICT \& Future Planning (2016R1A2B301306115) and Ministry of Trade, Industry \& Energy, Republic of Korea (P0013915). Disclosures: The authors declare no conflicts of interest.

\section{References}

1. W. Denk, J. Strickler, and W. Webb, "Two-photon laser scanning fluorescence microscopy," Science 248, 73-76 (1990).

2. H. Bao et al. "Fast handheld two-photon fluorescence microendoscope with a $475 \mu \mathrm{m} \times$ $475 \mu \mathrm{m}$ field of view for in vivo imaging," Opt. Lett. 33, 1333-1335 (2008).

3. D. Y. Kim et al. "Lissajous scanning two-photon endomicroscope for in vivo tissue imaging," Sci. Rep. 9(1), 3560 (2019).

4. A. Dilipkumar et al., "Label-free multiphoton endomicroscopy for minimally invasive in vivo imaging," Adv. Sci. 6(8), 1801735 (2019).

5. D. Huang et al., "Optical coherence tomography," Science 254, 1178-1181 (1991).

6. H.-C. Park et al., "Forward imaging OCT endoscopic catheter based on MEMS lens scanning," Opt. Lett. 37, 2673-2675 (2012).

7. B. J. Vakoc et al., "Cancer imaging by optical coherence tomography: preclinical progress and clinical potential," Nat. Rev. Cancer 12, 363 (2012).

8. B. R. Masters, "Book review: Handbook of biological confocal microscopy, Second Edition, Edited by J. B. Pawley," Opt. Eng. 35, 1 (1996).

9. P.-L. Hsiung et al., "Detection of colonic dysplasia in vivo using a targeted heptapeptide and confocal microendoscopy," Nat. Med. 14, 454 (2008).

10. G. Li et al., "Ultra-compact microsystems-based confocal endomicroscope," IEEE Trans. Med. Imaging 39(7), 2406-2414 (2020).

11. D. Wang et al., "Correction of image distortions in endoscopic optical coherence tomography based on two-axis scanning MEMS mirrors," Biomed. Opt. Express 4, 2066-2077 (2013).

12. C. Duan et al., "An endoscopic forward-viewing OCT imaging probe based on a two-axis scanning MEMS mirror," in IEEE 11th Int. Symp. Biomed. Imaging (ISBI), pp. 1397-1400 (2014).

13. J. Sun et al. "3D In Vivo optical coherence tomography based on a low-voltage, large-scanrange 2D MEMS mirror," Opt. Express 18, 12065-12075 (2010).

14. W. Liang et al., "Increased illumination uniformity and reduced photodamage offered by the Lissajous scanning in fiber-optic two-photon endomicroscopy," J. Biomed. Opt. 17, 021108 (2012).

15. D. Do, H. Yoo, and D. G. Gweon, "Fiber-optic raster scanning two-photon endomicroscope using a tubular piezoelectric actuator," J. Biomed. Opt. 19, 066010 (2014).

16. E. J. Seibel and Q. Y. J. Smithwick, "Unique features of optical scanning, single fiber endoscopy," Lasers Surg. Med. 30, 177-183 (2002).

17. N. Zhang et al., "Compact piezoelectric transducer fiber scanning probe for optical coherence tomography," Opt. Lett. 39, 186-188 (2014).

18. C. M. Lee et al., "Scanning fiber endoscopy with highly flexible, $1 \mathrm{~mm}$ catheterscopes for wide-field, full-color imaging," J. Biophotonics 3, 385-407 (2010).

19. D. Lorenser, R. A. McLaughlin, and D. D. Sampson, "Optical coherence tomography in a needle format," Optical Coherence Tomography in a Needle Format, pp. 2413-2472 (2015). 
20. D. R. Rivera et al., "Use of a lensed fiber for a large-field-of-view, high-resolution, fiberscanning microendoscope," Opt. Lett. 37(5), 881-883 (2012).

21. S. Kato, S. Chonan, and T. Aoki, "High-numerical-aperture microlensed tip on an air-clad optical fiber," Opt. Lett. 39(4), 773-776 (2014).

22. E. J. Min et al., "Single-body lensed-fiber scanning probe actuated by magnetic force for optical imaging," Opt. Lett. 34(12), 1897-1899 (2009).

23. H. C. Park, Y. H. Seo, and K. H. Jeong, "Lissajous fiber scanning for forward viewing optical endomicroscopy using asymmetric stiffness modulation," Opt. Express 22, 5818-5858 (2014).

24. K. Hwang, Y. H. Seo, and K. H. Jeong, "Microscanners for optical endomicroscopic applications," Micro Nano Syst. Lett. 5(1), 1-11 (2017).

25. K. Hwang et al., "Frequency selection rule for high definition and high frame rate Lissajous scanning," Sci. Rep. 7, 14075 (2017).

26. H. Kogelnik, "On the propagation of Gaussian beams of light through lens like media including those with a loss or gain variation," Appl. Opt. 4, 1562-1569 (1965).

27. W. Jung et al., Numerical Analysis of Gradient Index Lens-Based Optical Coherence Tomography Imaging Probes, p. 10, SPIE Press (2010).

28. H. C. Park et al., "Micromachined tethered silicon oscillator for an endomicroscopic Lissajous fiber scanner," Opt. Lett. 39, 6675-6678 (2014).

29. K. Hwang et al., "Handheld endomicroscope using a fiber-optic harmonograph enables realtime and in vivo confocal imaging of living cell morphology and capillary perfusion," Microsyst. Nanoeng. 6(1), 1-11 (2020).

Jae-Beom Kim received his $\mathrm{PhD}$ in bio and brain engineering a KAIST, Republic of Korea, in 2018. Currently, he is a senior engineer at SK Hynix in the Republic of Korea.

Jaehun Jeon received his BSc degree in bio and brain engineering from KAIST, Republic of Korea, in 2020. Currently, he is an MS student in bio and brain engineering from KAIST.

Kyungmin Hwang received her $\mathrm{PhD}$ in bio and brain engineering from KAIST, Republic of Korea, in 2020. Currently, she serves as a CEO at VPIX Medical, Inc. in the Republic of Korea.

Daniel Y. Kim received his MS degree in bio and brain engineering from KAIST, Republic of Korea, in 2018. He is currently studying at the School of Medicine from Cha University, Republic of Korea.

Ki-Hun Jeong received his PhD from the University of California at Berkeley in 2005. Currently, he is a full professor and the department head in the Department of Bio and Brain Engineering at KAIST in the Republic of Korea, as well as a codirector at KAIST Institute for Health Science and Technology. 